\title{
Antimalarials: Pre-clinical development update
}

\author{
Moumita Bala ${ }^{1}$, Lahiry Sandeep ${ }^{2}$, Shouvik Choudhury ${ }^{3}$, Ayan Mukherjee ${ }^{4}$, \\ Kiran Bhunya ${ }^{5}$, Koustuv Chowdhury ${ }^{6}$, Rajasree Sinha ${ }^{7}$ \\ 1-6Post Graduate Trainee, Department of Pharmacology, Institute of Post Graduate Medical Education and Research, \\ Kolkata, ${ }^{7}$ Post Graduate Trainee, Department of Pediatrics, Medical College, Kolkata
}

\section{A B S T R A C T}

Diverse strategies exist for the development of novel antimalarials to tackle the increasing incidence of artemisin resistance. New molecules have been developed which hold a promise to cater to this unmet need. We discuss few drugs currently under pre-clinical development that have shown encouraging results.

Key words: Artemisin, Resistance, Malaria, Novel drugs
Access this article online

Website:

http://nepjol.info/index.php/AJMS

DOI: 10.3126/ajms.v8i5.17530

E-ISSN: 2091-0576

P-ISSN: 2467-9100

\section{INTRODUCTION}

The challenge of the antimalarial drug development is to deliver a drug that should: (i) address drug-resistance issues, (ii) have a rapid onset of action, (iii) be safe, especially in children and pregnant women, and (iv) cure malaria in a single dose (v) identify novel targets. Artemisinin-based combination therapies (ACTs) are currently gold standard in treating uncomplicated malaria, have an inherent issue of emerging resistance. To tackle this problem, sophisticated cellular and phenotypic screening methods have identified drug candidates active against different stages of the parasites life cycle in recent years (Table 1). We discuss few of such innovative strategies currently under preclinical development.

\section{PRE CLINICAL DEVELOPMENT UPDATE}

The mitochondrial enzyme dihydroorotate dehydrogenase (DHOD) has been recognized as a potential antimalarial target, as it catalyzes the fourth step in the essential de novo pyrimidine biosynthesis pathway. ${ }^{1,2} \mathrm{~A}$ new chemical class, the triazolopyrimidines, has been identified with potent activity in whole-cell assays ( $P$. falciparum $\mathrm{IC}_{50}=79 \mathrm{nM}$ ) and $>5000$ fold specificity for parasite over human DHOD, though this class was inactive in the $P$. berghei in vivo model. The molecules underwent series of chemical modifications, and a potent lead discovered that had an $\mathrm{IC}_{50}$ of $40-50 \mathrm{nM}$ against drugsensitive and drug-resistant P. falciparum, including those resistant to chloroquine, atovaquone, and the antifolate, pyrimethamine. ${ }^{3}$ A drug canididate, DSM265, showed similar potency as chloroquine in mouse models, and is now the first DHOD inhibitor to enter pre-clinical trials. ${ }^{4}$

In-silico molecular docking approach was used to identify potential inhibitors that disrupt the interaction between the carboxy terminal tail of myosin $\mathrm{A}$ and the myosin tail interacting protein (MTIP) of the malaria parasite. This interaction is required for erythrocyte invasion. Optimization of a urea-pyrazole scaffold has yielded a molecule, 21A092, which targets the protein, has now advanced to preclinical studies. ${ }^{5}$

Synthetic peroxides are proving to be useful substitutes for artemisinin. The first-generation ozonide OZ277, known as arterolane, has been found to inhibit the growth of chloroquine-resistant (K1) and chloroquine sensitive (NF54) parasite strains with an IC50 $=1.6-1.8 \mathrm{nM}$. In 2012, the combination of arterolane maleate and piperaquine phosphate was released as a 3-day treatment in India. The second-generation peroxide OZ439 


\section{Table 1: Summary of novel antimalarial compounds under preclinical development}

\begin{tabular}{llll}
\hline Compound & Chemical class & Target or MOA & Therapeutic activity \\
\hline DSM265 & Triazolopyrimidine & DHOD & Chemotherapeutic \\
21 A092 & Pyrazole & Unknown & Chemotherapeutic \\
MMV390048 & Aminopyridine & Unknown & Chemotherapeutic \\
P218 & Diaminopyridine & DHFR & Chemotherapeutic; Prophylactic \\
RKA182 & 1,2,4,5-tetraoxane & Hemoglobin digestion & Chemotherapeutic \\
ELQ-300 & Quinolone-3-Diarylether & Cytochrome bc1 & Chemotherapeutic; Transmission blocking; Prophylactic \\
BCX4945 & Immucillin-G & PNP & Chemotherapeutic \\
NPC-1161B & 8-aminoquinoline & Unknown & Chemotherapeutic; Transmission blocking; Prophylactic \\
\hline DHOD: Dihydroorotatereductase; DHFR: Dihydrofolatereductase; PNP: Purine nucleoside phosphatase; MOA: Mechanism of action
\end{tabular}

$(\mathrm{EC} 50=3.4-4.0 \mathrm{nM})$ featuring an 80- aryl rather than an 80-alkyl group causing higher stability of the O-O bond towards Fe(II) increasing by 50-fold, presumably because of steric reasons, is now in Phase IIa studies. ${ }^{6,7}$ Tetraoxanes (also stabilizes $\mathrm{O}-\mathrm{O}$ bond), as was employed in the drug development candidate RKA 182, has displayed IC50 values of $4.9 \mathrm{nM}$ against the P. falciparum 3D 7 strain and of $1.9 \mathrm{nM}$ against the $\mathrm{K} 1$ strain (chloroquine sensitive and -resistant, respectively). It reduced $P$. berghei parasitemia in mice to undetectable levels 24 hours after treatment. This compound is currently in preclinical trials and although it has greater stability than OZ277, its antimalarial activity is inferior to OZ439. ${ }^{8,9}$

Phenotypic whole-cell screening method has revealed a 2-aminopyridine class of small molecules as a good starting point to develop new a derivative, MMV390048, which lacked cross-resistance with current drugs used to treat malaria, and has a potential ability to block all life cycle stages of the malaria parasite. Both genomic and chemoproteomic studies have identified a kinase of the Plasmodium parasite, phosphatidylinositol 4-kinase, as the molecular target of MMV390048. This compound was efficacious against all Plasmodium life cycle stages, apart from late hepatic hypnozoites. Efficacy was shown in the humanized Plasmodium falciparum mouse model, and modest reductions in mouse-to-mouse transmission were achieved in the Plasmodium berghei mouse model. ${ }^{10,11}$

P218, a dihydrofolatereducatse inhibitor (DHFR) inhibitor has been found to be active against all clinically relevant mutations of the parasite. It combines the pyrimidine ring of pyrimethamine which brings potency, and the linker of the DHFR inhibitor WR99210, which tolerates mutations due to its flexibility. P218 is more potent than pyrimethamine against DHFR in the wild-type strain TM4 (IC50 = 4.6 and $58 \mathrm{nM}$, respectively) as well as in the quadruple mutant strain V1/S (IC50 = 56 and $>100,000 \mathrm{nM}$, respectively). It has an activity against quadruple mutant P. falciparum in mice, with an ED50 = $0.3 \mathrm{mg} / \mathrm{kg} /$ day, orally. ${ }^{12,13}$
The 8-aminoquinoline (8AQ) class of anti-malarial compounds is unique due to the efficacy against relapsing forms of malaria. This activity is a result of the antihypnozoite activity. Paired along with the gametocidal activity of primaquine (PQ), 8AQs make a class attractive for mass administration in efforts towards malaria eradication.Tafenoquine (TQ) is an 8-aminoquinoline (8AQ) currently in late stage development as an antimalarial prophylactic agent. NPC-1161B is another promising $8 \mathrm{AQ}$ in late preclinical development. ${ }^{14,15}$

ELQ-300 is an endochin-like quinolone and the first in a new class of antimalarials known as quinolone-3diarylethers. It acts as an inhibitor of the mitochondrial cytochrome bc1 complex (complex III in the electron transport chain). In preclinical studies with mice, it was found to be highly active against all life cycle stages of the malaria parasite. It targets the liver and blood stages of Plasmodium falciparum, as well as the forms that are crucial to transmission of disease: gametocytes, zygotes, and ookinetes. ${ }^{16,17}$

Blocking of purine nucleoside phosphorylase (PNP) has been found to kill cultured parasites by purine starvation, since Plasmodium species are known to be purine auxotrophs. DADMe-Immucillin-G (BCX4945) is a transition state analogue of human and Plasmodium PNPs, binding with picomolar affinity. Metabolite analysis has demonstrated that PNP blockade inhibits purine salvage and polyamine synthesis in the parasites. The efficacy, oral availability, chemical stability, unique mechanism of action and low toxicity of BCX4945 demonstrate potential for combination therapies with this novel antimalarial agent. $^{18,19}$

\section{CONCLUSION}

It is an exciting time for malaria drug discovery, and the drug pipeline looks robust. The combination of new and innovative screens to identify compounds with broad-range activity is hoped to yield new insight into chemicals with broad-range activity. 


\section{REFERENCES}

1. Ittarat I, Asawamahasakda W and Meshnick SR. The effects of antimalarials on the Plasmodium falciparum dihydroorotate dehydrogenase. ExpParasitol 1994;79:50-56.

2. Reyes P, Rathod PK, Sanchez DJ, Mrema JE, Rieckmann KH and Heidrich HG. Enzymes of purine and pyrimidine metabolism from the human malaria parasitePlasmodium falciparum. MolBiochemParasitol 1982;5(5):275-290.

3. Gujjar R, Marwaha A, El Mazouni F, White J, White KL, Creason $\mathrm{S}$, et al. Identification of a metabolically stable triazolopyrimidine-based dihydroorotate dehydrogenase inhibitor with antimalarial activity in mice. Journal of medicinal chemistry 2009;52:1864-1872.

4. Phillips MA, Lotharius J, Marsh K, White J, Dayan A, White KL, et al. A long-duration dihydroorotate dehydrogenase inhibitor (DSM265) for prevention and treatment of malaria. Science translational medicine 2015;7(296):296ra111.

5. NC Wells T. Discovering and developing new medicines for malaria control and elimination. Infectious Disorders-Drug Targets (Formerly Current Drug Targets-Infectious Disorders). 2013;13:292-302.

6. Wang X, Dong Y, Wittlin S, Charman SA, Chiu FC, Chollet J, et al. Comparative antimalarial activities and ADME profiles of ozonides (1, 2, 4-trioxolanes) OZ277, OZ439, and their 1 , 2-dioxolane, 1, 2, 4-trioxane, and 1, 2, 4, 5-tetraoxane isosteres. Journal of medicinal chemistry 2013;56:2547-2555.

7. Biamonte MA, Wanner $\mathrm{J}$ and Le Roch KG. Recent advances in malaria drug discovery. Bioorganic \& medicinal chemistry letters 2013;23(10):2829-2843.

8. Kumar N, Khan SI, Rajalakshmi G, Kumaradhas P and Rawat DS. Synthesis, antimalarial activity and cytotoxicity of substituted 3, 6-diphenyl-[1, 2, 4, 5] tetraoxanes. Bioorganic \& medicinal chemistry 2009;17:5632-5638.

9. O'Neill PM, Amewu RK, Nixon GL, BousejraEIGarah F, Mungthin $\mathrm{M}$, Chadwick $\mathrm{J}$, et al. Identification of a 1, 2, 4, 5-Tetraoxane Antimalarial Drug-Development Candidate (RKA 182) with Superior Properties to the Semisynthetic Artemisinins. Angewandtechemie international edition 2010;49:5693-5697.

10. Ghidelli-Disse S, Lafuente-Monasterio MJ, Waterson D, Witty M,
Younis Y, Paquet T, et al. Identification of Plasmodium PI4 kinase as target of MMV390048 by chemoproteomics. Malaria Journal 2014;13: 38

11. Paquet T, Le Manach C, Cabrera DG, Younis $Y$, Henrich PP, Abraham TS, et al. Antimalarial efficacy of MMV390048, an inhibitor of Plasmodium phosphatidylinositol 4-kinase. Science translational medicine 2017;9:eaad9735.

12. Abbat $S$, Jain $V$ and Bharatam $P V$. Origins of the specificity of inhibitor P218 toward wild-type and mutant PfDHFR: A molecular dynamics analysis. Journal of Biomolecular Structure and Dynamics 2015;33:1913-1928.

13. Yuthavong $\mathrm{Y}$, Tarnchompoo $\mathrm{B}$, Vilaivan $\mathrm{T}$, Chitnumsub $\mathrm{P}$, Kamchonwongpaisan S, Charman SA, et al. Malarial dihydrofolatereductase as a paradigm for drug development against a resistance-compromised target. Proceedings of the National Academy of Sciences 2012;109:16823-16828.

14. Marcsisin SR, Sousa JC, Reichard GA, Caridha D, Zeng Q, Roncal N, et al. Tafenoquine and NPC-1161B require CYP 2D metabolism for anti-malarial activity: implications for the 8 -aminoquinoline class of anti-malarial compounds. Malaria journal 2014;13:2.

15. Duffy $S$ and Avery VM. Identification of inhibitors of Plasmodium falciparum gametocyte development. Malaria journal 2013;12:408.

16. Miley GP, PouS, Winter R, Nilsen A, Li Y, Kelly JX, et al. ELQ300 prodrugs for enhanced delivery and single-dose cure of malaria. Antimicrobial agents and chemotherapy 2015;59:55555560.

17. Nilsen A, LaCrue AN, White KL, Forquer IP, Cross RM, Marfurt J, et al. Quinolone-3-diarylethers: a new class of antimalarial drug. Science translational medicine 2013;5(177):177ra37.

18. Suthar MK, Verma A, Doharey PK, Singh SV and Saxena JK. Single tryptophan of disordered loop from plasmodium falciparum purine nucleoside phosphorylase: involvement in catalysis and microenvironment. Applied biochemistry and biotechnology 2013;170:868-879.

19. Zhang Y, Evans GB, Clinch K, Crump DR, Harris LD, Fröhlich RF, et al. Transition state analogues of Plasmodium falciparum and human orotatephosphoribosyltransferases. Journal of Biological Chemistry. 2013;288:34746-34754.

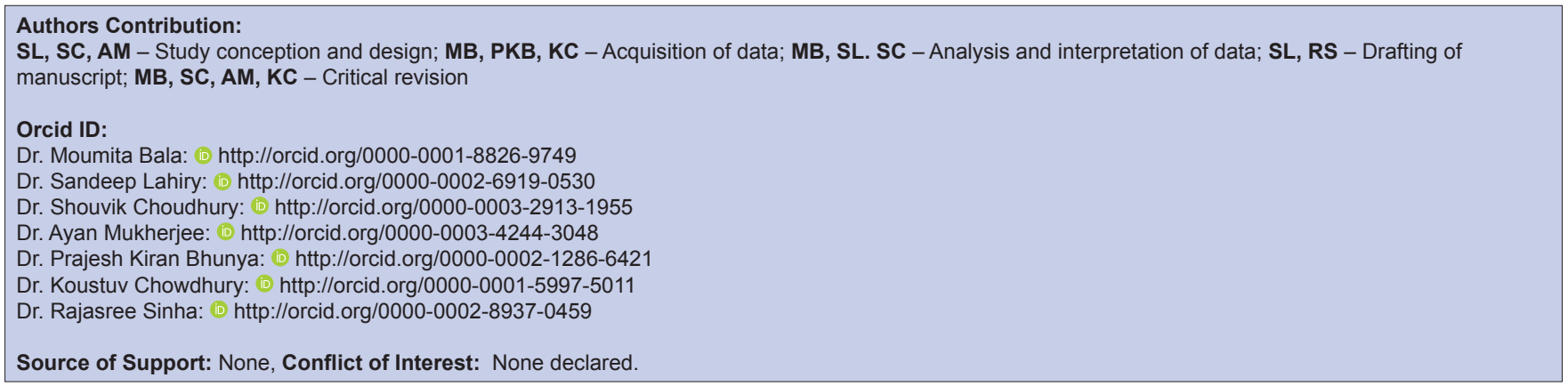

